Original Article

\title{
A Possible contributor to Erythropoiesis-Stimulating Agents Requirement in Hemodialysis: Paraoxonase 1 Activity
}

\author{
Berfu KORUCU ${ }^{1}$ (D) , Hasan YETER ${ }^{1}$ (D) , Elif Burcu BALI² (i) , Mehmet Kursat DERICI ${ }^{3}$ (iD) , \\ Galip GUZ ${ }^{1}$
}

${ }^{1}$ Department of Nephrology, Gazi University Faculty of Medicine, Ankara, Turkey

${ }^{2}$ Gazi University Vocational School of Health Services, Ankara, Turkey

${ }^{3}$ Department of Pharmacology, Kirikkale University Faculty of Medicine, Kirikkale, Turkey

\section{A B S T R A C T}

Background: Anemia is one of the most prevalent complications of chronic kidney disease. In chronic kidney disease-related anemia, circulating erythrocytes have a decreased life span. Paraoxonase-1 activity is one of the essential factors protecting cell membranes, e.g., erythrocytes, from lipid peroxidation under normal conditions. In this study, we investigated Paraoxonase- 1 activity in hemodialysis patients with and without erythropoiesis-stimulating agent therapy and the possible relationship of Paraoxonase-1 activity levels with the erythropoiesis-stimulating agent dose required.

Material and Methods: This study is composed of patients undergoing hemodialysis with a minimum dialysis vintage of six months. We excluded cases with an active infection, cardiovascular events and malignancy, hypo- or hyperthyroidism, central venous catheters, iron deficiency, cystic kidney diseases, nephrectomy, and routine C-reactive protein levels higher than reference range within the last six months. Results: Baseline characteristics, laboratory parameters, oxidative stress, and systemic inflammatory indices were similar between groups. The mean Paraoxonase-1 activity of the erythropoiesis-stimulating agent+group was significantly lower than the erythropoiesis-stimulating agent-group (191.4 \pm 118.8 and 488.1 \pm 174.9 , respectively; $\mathrm{p}<0.001)$. Cumulative erythropoiesis-stimulating agent doses and Paraoxonase- 1 activity were significantly and negatively correlated $(R=-0.736, p<0.001)$.

Conclusions: The results of this study suggest that lower Paraoxonase-1 activity in hemodialysis patients is associated with the requirement for erythropoiesis-stimulating agent therapy, presumably due to decreased life span of erythrocytes. Further studies examining the relationship between Paraoxonase-1 activity and erythropoiesis-stimulating agent treatment requirements are necessary to reveal new treatment goals for chronic kidney disease-related anemia.

Turk J Int Med 2021

DOI: $\underline{10.46310 / \text { tjim. } 882813}$

Keywords: Hepatitis B, Rheumatoid arthritis, Parenteral therapies.

Addressfor Correspondence:
Berfu Korucu MD




\section{Introduction}

Anemia is one of the most prevalent complications of chronic kidney disease (CKD) ${ }^{1,2}$ In CKD related anemia, circulating erythrocytes have a decreased life span, and compensatory erythropoiesis is decreased. ${ }^{3}$ In the latter, the most prominent mechanism is the reduction in intrinsic erythropoietin (EPO) secretion. However, the secretion of EPO remains significant even in end-stage renal disease in patients without total nephrectomy. ${ }^{4}$ The primary condition that requires the use of erythropoiesis-stimulating agents (ESA) is patients' blunt response to low hemoglobin $(\mathrm{Hb})$ level. ${ }^{5}$ On the other hand, every patient's need for ESA treatment is not the same, and even some patients persistently have adequate $\mathrm{Hb}$ levels without ESA.

The pathophysiological mechanism of decreased erythrocyte life span in CKD patients has not been clarified. ${ }^{6}$ Studies investigating the possible mechanical effects of hemodialysis have failed to reveal significant differences with peritoneal dialysis patients. ${ }^{7}$ The so-called "uremic milieu" in CKD describes numerous pathophysiological mechanisms such as increased uremic toxins, systemic inflammation, and oxidative stress. ${ }^{8}$ On the other hand, there is an inadequacy in the mechanisms that balance these phenomena.

Among the components of the "uremic milieu," one of the significant pathophysiological mechanisms that concern cellular life span is the increased cell membrane lipid peroxidation in chronic renal failure. ${ }^{9}$ Paraoxonase-1 (PON1) activity in high-density lipoprotein (HDL) is one of the essential factors protecting cell membranes, e.g., erythrocytes, from lipid peroxidation under normal conditions. ${ }^{10,11}$ The protective effect of PON1 on the erythrocyte membrane is observed in animal models and human studies. ${ }^{12,13}$

Studies have shown that patients with CKD have decreased PON1 activity compared to the healthy population, and revealed its adverse outcomes on patient survival. ${ }^{14,15}$ In this study, we investigated PON1 activity in HD patients with and without ESA therapy, excluding cases with total nephrectomy or cystic kidney diseases, anemia not related to CKD, and factors that may cause ESA resistance. We also analyzed the possible relationship of PON1 activity levels with the ESA dose required.

\section{Material and Methods}

\section{Statement of Ethics}

The study protocol was approved by an independent research institute's committee on human research.

\section{Study Design, Participants, and Definitions}

This cross-sectional study is composed of 50 patients undergoing $\mathrm{HD}$ with a minimum dialysis vintage of six months. We excluded cases with an active infection, an active or recent history of any cardiovascular events and malignancy, hypo- or hyperthyroidism, central venous catheters, iron deficiency, prosthetic heart valves, unilateral or bilateral nephrectomy, polycystic kidney disease or acquired kidney cysts, routine C-reactive protein (CRP) levels higher than reference range within the last six months, a calculated erythropoietin resistance index (ERI) of $\geq 5 \mathrm{U} / \mathrm{kg} /$ week $/ \mathrm{g} / \mathrm{dL}$, and a single pool Kt/V of $\leq 1$.2. Informed consent was obtained from all patients for being included in the study.

The ERI was calculated as the weekly recombinant human erythropoietin (rhEPO) dose divided by the body weight and the hemoglobin concentration. ${ }^{16}$ Mean weekly and cumulative rhEPO doses applied within the last six months were calculated. Doses of darbepoetin were converted to equivalent doses of epoetin for standardization. ${ }^{17}$ Patients with $>100 \mathrm{~mL} /$ day urine output were considered to have a residual renal function (RRF). ${ }^{18}$ Single pool Kt/V calculated by Daugirdas' secondgeneration formula. Urea reduction ratio (URR) calculated by taking the difference between preand post-dialysis urea levels and divided by predialysis urea levels.

All of the patients treated with a standard HD procedure via A-V fistulae, each session lasted 4 hours, three times a week, using bicarbonatecontaining dialysate and low-flux polysulfone membrane. The blood flow rate ranged from 300 to $350 \mathrm{~mL} / \mathrm{min}$, and the dialysate flow rate was $500 \mathrm{~mL} / \mathrm{min}$. 


\section{Blood Specimen Collection}

Blood specimens were collected at the initiation of a mid-week session. Specimens were allowed to clot at room temperature for 30 minutes. Clot removed by centrifuging samples at $3500 \mathrm{rpm}$ for 10 minutes. The serum immediately transferred into a polypropylene tube and stored at $-80^{\circ} \mathrm{C}$.

\section{Materials and Measurements}

Total oxidant status (TOS) and total antioxidant status (TAS), high sensitive CRP (hsCRP), interleukin-6 (IL-6), and PON1 activity were measured from freshly collected serum. Last (within two weeks) blood urea nitrogen (BUN), creatinine (cre), albumin (alb), hemogram test, and lipid profile results were evaluated retrospectively. The mean value of the last six months was calculated for intact parathormone and ferritin levels. Patients' demographic features such as age, sex, and body mass index were noted.

\section{Biochemical Study}

Serum levels of hsCRP were studied by the nephelometric technique using $\mathrm{BN}$ II/BN ProSpec (Siemens, Marburg, Germany), and IL-6 were studied by chemiluminescence immunoassay technique using Immulite 2000 (Siemens Diagnostics, Gwynedd, UK).

Total oxidant status (TOS) and total antioxidant status (TAS) levels were measured using commercially available kits (RelAssay Diagnostics, Ankara, Turkey). The ratio of TOS to TAS accepted as the OS index (OSI). For calculation, the unit of TAS converted to $\mu \mathrm{mol} / \mathrm{L}$, and the OSI calculated according to the following formula: OSI (arbitrary unit) $=$ TOS $(\mu \mathrm{mol} \mathrm{H} 2 \mathrm{O} 2$ equivalent/L)/TAS ( $\mu$ mol Trolox equivalent/L).

PON1 activity was measured using commercially available kits (RelAssay, Ankara, Turkey). The rate of paraoxon hydrolysis (diethylpnitrophenylphosphate) was measured by monitoring the increase of absorption at $412 \mathrm{~nm}$ at $37^{\circ} \mathrm{C}$. The amount of generated p-nitrophenol was calculated from the molar absorption coefficient at $\mathrm{pH} 8.5$, which was $18.290 \mathrm{M}^{-1} \mathrm{~cm}^{-1}$. PON1 activity was expressed as U/L.

\section{Statistical Methods}

Patients were grouped as "ESA+" and "ESA-" according to rhEPO or darbepoetin requirements within the last six months. Numeric values of the study were shown with mean and standard deviation or medians with ranges, categorical data, frequency, and percentage. For data normally disturbed, Unpaired Student's T-Test is used for comparison between two groups. For abnormal distributed data Kruskal-Wallis test performed. Pearson correlation test was used to find a correlation between continuous variables.

A p-value of less than 0.05 was considered statistically significant. All statistical analyses were performed using the Statistical Package for Social Science (SPSS, Chicago, IL, USA) for personal computers, version 21.0.

\section{Results}

\section{Baseline Characteristics}

The demographic characteristics, laboratory parameters including hemoglobin, ferritin and HDL, six months cumulative iron doses, dialysis adequacy calculations, HD vintages, and RRFs of patients in the ESA+ and ESA- groups were similar (Table 1).

\section{Systemic Inflammation and Oxidative Stress Indices of the Groups}

The mean hsCRP and IL-6 levels of patients in the ESA+ and ESA- groups were similar. Similarly, the TOS, TAS, and calculated OSIs of the groups were similar. The mean PON1 activity of the ESA+ group was significantly lower than the ESA- group (191.4 \pm 118.8 and $488.1 \pm 174.9$, respectively; $p<0.001$ ) (Table 2).

\section{Correlations of PON1 Activity with Patient-Related} Factors, Oxidative Stress, and Cumulative ESA Doses

There was no significant correlation between age, BMI, dialysis time, HDL level or OSI, and PON1 activity, as shown in Table 3 and Figure 1. Cumulative ESA doses and PON1 activity were significantly and negatively correlated $(R=-0.736, p<0.001)$.

\section{Discussion}

The results of our study suggest that the serum PON1 activity is significantly lower in $\mathrm{HD}$ patients requiring ESA therapy, and PON1 activity significantly correlates with cumulative 
Table 1. Baseline characteristics and laboratory parameters of the groups

\begin{tabular}{|c|c|c|c|}
\hline & $\mathbf{E S A}+(\mathbf{n}=32)$ & ESA- $(n=18)$ & $p$-value \\
\hline Female/male (n) & $14 / 18$ & $5 / 13$ & 0.273 \\
\hline Age mean (SD) & $58.6( \pm 14.7)$ & $55.1( \pm 16.3)$ & 0.437 \\
\hline BMI, kg/m² (SD) & $23.4( \pm 4.9)$ & $25.6( \pm 5.1)$ & 0.150 \\
\hline Smoking, n (\%) & $6(18.8 \%)$ & $4(22.2 \%)$ & 0.774 \\
\hline Diabetes, n (\%) & $7(21.8 \%)$ & $5(27.7 \%)$ & 0.647 \\
\hline BUN mean, mg/dL (SD) & $67.1( \pm 16.2)$ & $57.6( \pm 15.7)$ & 0.067 \\
\hline Creatinine mean, mg/dL (SD) & $8.4( \pm 1.7)$ & $8.1( \pm 2.0)$ & 0.543 \\
\hline Hemoglobin mean, g/dL (SD) & $11.3( \pm 1.2)$ & $11.2( \pm 2.0)$ & 0.831 \\
\hline Albumin mean, g/dL (SD) & $3.8( \pm 0.3)$ & $3.8( \pm 0.3)$ & 0.837 \\
\hline iPTH median, pg/mL (min-max) & $314.5(14-1834)$ & $296(69.2-1401.1)$ & 0.693 \\
\hline Ferritin mean, ng/mL (SD) & $568.6( \pm 174.7)$ & $499.5( \pm 281.0)$ & 0.289 \\
\hline LDL mean, mg/dL (SD) & $107.7( \pm 42.4)$ & $101.0( \pm 35.1)$ & 0.570 \\
\hline Triglyceride mean, mg/dL (SD) & $204.0( \pm 140.6)$ & $184.0( \pm 114.7)$ & 0.626 \\
\hline HDL mean, mg/dL (SD) & $38.6( \pm 8.9)$ & $38.3(10.8)$ & 0.916 \\
\hline IV iron median, mg (min-max) & $600(0-2200)$ & $600(0-1200)$ & 0.074 \\
\hline ACEI/ARB use, $n(\%)$ & $5(15.6 \%)$ & $2(11.1 \%)$ & 0.303 \\
\hline $\mathrm{Kt} / \mathrm{V}$ mean $(\mathrm{SD})$ & $1.6( \pm 0.3)$ & $1.6( \pm 0.4)$ & 0.880 \\
\hline URR (\%) mean (SD) & $75.2( \pm 6.2)$ & $74.6( \pm 7.0)$ & 0.747 \\
\hline HD vintage mean, months (SD) & $70.6( \pm 56.7)$ & $62.8( \pm 48.3)$ & 0.629 \\
\hline RRF, n (\%) & $11(34.3 \%)$ & $6(33.3 \%)$ & 0.942 \\
\hline
\end{tabular}

ESA: erythropoiesis-stimulating agent, BMI: body mass index, BUN: blood urea nitrogen, iPTH: intact parathormone, LDL: low-density lipoprotein, HDL: high-density lipoprotein, ACEI/ARB: angiotensin-converting enzyme inhibitors/ angiotensin receptor blockers, URR: urea reduction ratio, HD: hemodialysis, RRF: residual renal function

Table 2. Systemic inflammation and oxidative stress indices of the groups

\begin{tabular}{llll}
\hline & ESA $+(\mathrm{n}=32)$ & ESA- $(\mathrm{n}=18)$ & p-value \\
\hline hsCRP mean, mg/L (SD) & $5.20( \pm 2.4)$ & $4.34( \pm 2.2)$ & 0.223 \\
IL-6 mean, pg/mL (SD) & $3.8( \pm 0.9)$ & $3.7( \pm 0.9)$ & 0.791 \\
TOS mean, $\mu \mathrm{mol} / \mathrm{L}(\mathrm{SD})$ & $3.22( \pm 2.3)$ & $3.14( \pm 2.5)$ & 0.917 \\
TAS mean, $\mu \mathrm{mol} / \mathrm{L}(\mathrm{SD})$ & $2.33( \pm 0.2)$ & $2.31( \pm 0.3)$ & 0.747 \\
OSI & $0.13( \pm 0.1)$ & $0.14( \pm 0.1)$ & 0.970 \\
PON1 mean, U/L (SD) & $191.4( \pm 118.8)$ & $488.1( \pm 174.9)$ & $<0.001$ \\
\hline
\end{tabular}

hsCRP: high sensitive C-reactive protein, IL-6:interleukin-6, TOS: total oxidant status, TAS: total antioxidant status, OSI: oxidative stress index, PON1: paraoxonase-1 activity 
Table 3. Correlations of PON1 with patient-related factors, oxidative stress, and cumulative ESA doses

PON1 activity (U/L)

\begin{tabular}{lcc} 
& $\mathrm{R}$ & $\mathrm{p}$-value \\
\hline Age & -0.084 & 0.560 \\
BMI, kg/m² & -0.031 & 0.831 \\
HD vintage & 0.023 & 0.872 \\
HDL (mg/dL) & $-0,132$ & 0.361 \\
OSI & -0.072 & 0.618 \\
Cumulative ESA dose (U) & $-0,736$ & $<0.001$ \\
\hline
\end{tabular}

PON1: paraoxonase-1, BMI: body mass index, HD: hemodialysis, HDL: high-density lipoprotein, ESA: erythropoiesis-stimulating agent

Figure 3. Correlations of PON1 with patient-related factors, oxidative stress indices and cumulative ESA doses (PON1: paraoxonase-1, BMI: body mass index, HD: hemodialysis, HDL: high-density lipoprotein, ESA: erythropoiesis-stimulating agent)
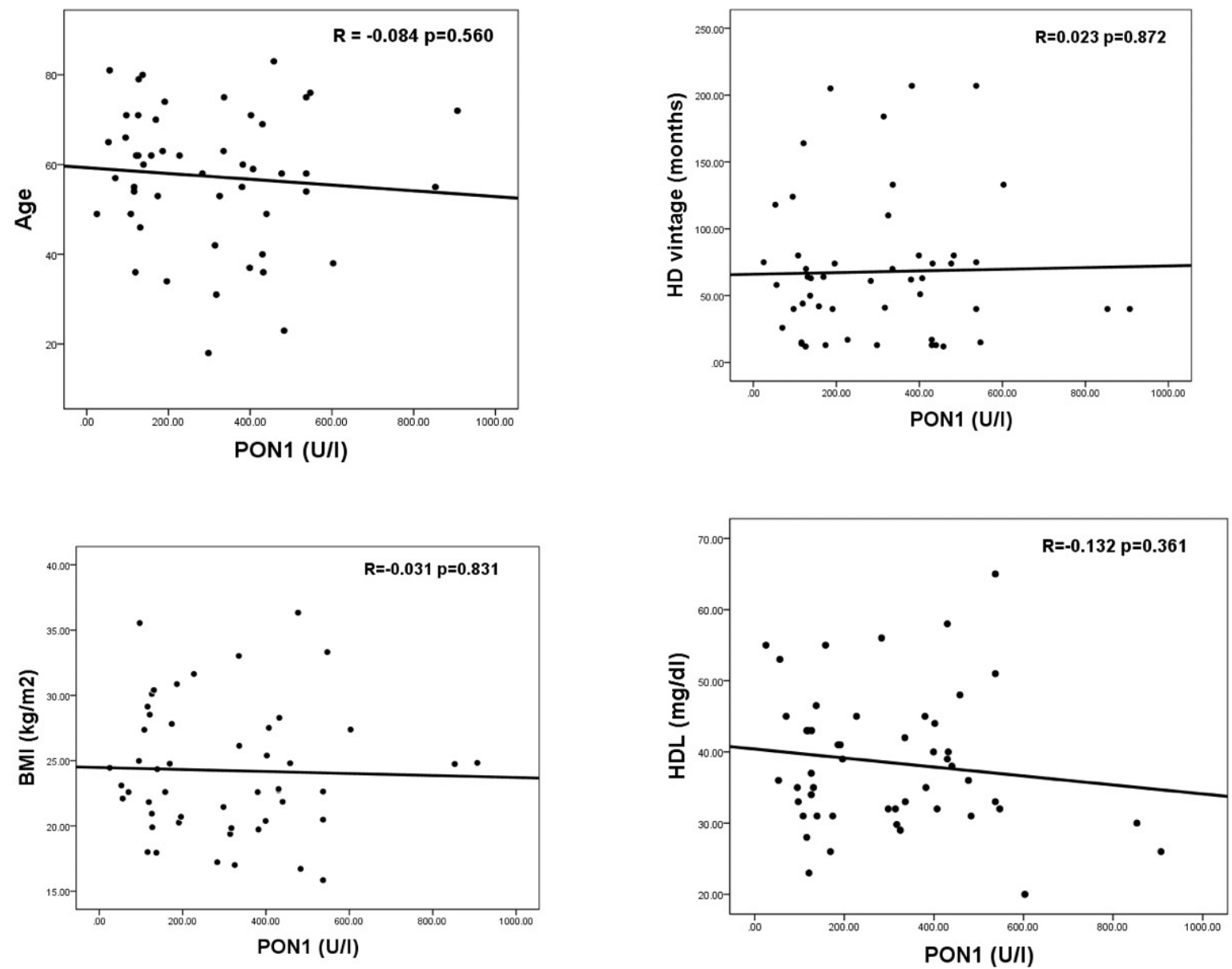

ESA doses. PON1 activity is not associated with age, BMI, serum HDL levels, dialysis vintage, and oxidative stress index.

Decreased erythrocyte life span is one of the major determinants of CKD related anemia. Median erythrocyte life span decreased by $20 \%$

in HD patients compared with healthy controls. ${ }^{19}$ However, the reason for this significant decrease is not well understood. In one study, IL-6, IL-18, IL-10, and hsCRP levels were compared with the erythrocyte life span, but no association was found. ${ }^{20}$ On the other hand, in this study, 
erythrocyte life span and uric acid were positively correlated. This finding suggests that antioxidant mechanisms may have a role in this subject.

HDL is a lipoprotein that binds highdensity cholesterol, takes part in the transport of cholesterol from the periphery to the liver, and is inversely associated with cardiovascular morbidities. ${ }^{21} \mathrm{HDL}$ is composed of apolipoprotein A-I and A-II and also many antioxidant proteins, including PON1. ${ }^{22}$ PON1 is now considered to be more prominent than HDL as the source of positive cardiovascular effects. ${ }^{23}$ The human PON1 gene is on chromosome 7 and encodes PON1, PON2, and PON3. ${ }^{24}$ PON1 is synthesized in the liver and transported to the periphery by HDL to perform hydrolysis of oxidized phospholipids on cellular membranes. ${ }^{25}$ These uniform features make PON1 one of the future treatment targets.

PON1 activity was found to be lower in HD patients compared to the healthy population and associated with adverse cardiovascular outcomes. ${ }^{26}$ Although there is no study directly examining the relationship between PON1 activity and anemia, there is evidence suggesting that its lower activity may also be associated with CKD related anemia. Riberio et al. investigated factors associated with PON1 activity level in $\mathrm{HD}$ patients and found lower activity in patients with systemic inflammation, longer dialysis vintage, and higher dose ESA therapy. ${ }^{27}$ Another study suggested that HD patients had increased hemolysis susceptibility of erythrocytes with lower PON1 activity. ${ }^{28}$

The possible cause of this significant PON1 activity difference in two similar $\mathrm{HD}$ groups may be due to PON1 gene polymorphisms. To date, two polymorphisms of PON1 and three polymorphisms of its promoter region was defined. ${ }^{29}$ However, the results of the studies are controversial in terms of the clinical significance of these polymorphisms. In addition, epigenetic factors have been reported to have an important effect on PON1 activity. ${ }^{30}$ Further studies are needed to clarify the effect of genetic and epigenetic factors on PON1 expression and activity in the HD population.

We designed our study to be suitable for understanding the relationship between PON1 activity and ESA treatment requirements in HD patients. To ensure this, we excluded all possible factors that contribute to the development of anemia in CKD patients. However, these strict exclusion criteria may have prevented us from observing factors that may affect PON1 activity levels. In order to confirm the results we obtained in our study, studies designed to directly examine the relationship between PON1 activity and erythrocyte life span in HD patients is needed. Thus, the pathophysiology of CKDrelated anemia will be better understood, and new targets for treatment can be introduced.

\section{Concluisons}

The results of this study suggest that lower PON1 activity in HD patients may be associated with the requirement for ESA therapy. Further studies examining the relationship between PON1 activity and ESA treatment requirements are necessary to reveal new treatment goals for CKD-related anemia.

\section{Conflict of Interest}

The authors declare that they have no conflict of interest.

\section{Acknowledgements}

All expenditures of the study were sponsored by the Internal Diseases Postgraduate Education Association, Turkey.

\section{Authors' Contribution}

Study Conception: BK; Study Design: BK, HY, EBB; Supervision: GG; Fundings: GG; Materials: BK, HY; Data Collection and/or Processing: BK, HY; Statistical Analysis and/or Data Interpretation: $\mathrm{BK}, \mathrm{HY}$; Literature Review: BK, HY, EBB, MKD; Manuscript Preparation: BK, HY; Critical Review: MKD, GG; Biochemical Measurements: EBB

\section{References}

1. Stauffer ME, Fan T. Prevalence of anemia in chronic kidney disease in the United States. PLoS One. 2014 Jan 2;9(1):e84943. doi: 10.1371/journal.pone.0084943.

2. Akizawa T, Okumura H, Alexandre AF, Fukushima A, Kiyabu G, Dorey J. Burden of Anemia in Chronic Kidney Disease Patients in Japan: A Literature Review. Ther Apher Dial. 2018 Oct;22(5):444-56. doi: 10.1111/1744-9987.12712. 
3. Zadrazil J, Horak P. Pathophysiology of anemia in chronic kidney diseases: A review. Biomed Pap Med Fac Univ Palacky Olomouc Czech Repub. 2015 Jun;159(2):197-202. doi: 10.5507/ bp.2013.093.

4. Wilmink J, Rosengarten P, Vet R, Goudsmit R. Serum erythropoietin (ESF) titers in anemia of chronic renal failure. J Lab Clin Med. 1982 Nov;100(5):720-34.

5. Ross RP, McCrea JB, Besarab A. Erythropoietin response to blood loss in hemodialysis patients in blunted but preserved. ASAIO J. 1994 Jul-Sep;40(3):M880-5. doi: 10.1097/00002480199407000-00122.

6. Brimble KS, McFarlane A, Winegard N, Crowther M, Churchill DN. Effect of chronic kidney disease on red blood cell rheology. Clin Hemorheol Microcirc. 2006;34(3):411-20.

7. Bissinger R, Artunc F, Qadri SM, Lang F. Reduced erythrocyte survival in uremic patients under hemodialysis or peritoneal dialysis. Kidney Blood Press Res. 2016;41(6):966-77. doi: 10.1159/000452600.

8. Glassock RJ. Uremic toxins: what are they? An integrated overview of pathobiology and classification. J Ren Nutr. 2008 Jan;18(1):2-6. doi: 10.1053/j.jrn.2007.10.003.

9. Korol L. Modeling in vitro pathways of activation of lipid peroxidation and protein in chronic kidney disease. Fiziol Zh. 2015;61(4):92-7. doi: 10.15407/fz61.04.092 (in Ukrainian).

10. Rosenblat M, Vaya J, Shih D, Aviram M. Paraoxonase 1(PON1) enhances HDL-mediated macrophage cholesterol efflux via the ABCA1 transporter in association with increased HDL binding to the cells: a possible role for lysophosphatidylcholine. Atherosclerosis. 2005 Mar;179(1):69-77. doi: 10.1016/j. atherosclerosis.2004.10.028.

11. Huang Y, Wu Z, Riwanto M, Gao S, Levison BS, Gu X, Fu X, Wagner MA, Besler C, Gerstenecker G, Zhang R, Li XM, DiDonato AJ, Gogonea V, Tang WH, Smith JD, Plow EF, Fox PL, Shih DM, Lusis AJ, Fisher EA, DiDonato JA, Landmesser U, Hazen SL. Myeloperoxidase, paraoxonase-1, and HDL form a functional ternary complex. J Clin Invest. 2013 Sep;123(9):3815-28. doi: 10.1172/JCI67478.

12. Ferretti G, Bacchetti T, Busni D, Rabini R, Curatola G. Protective effect of paraoxonase activity in high-density lipoproteins against erythrocyte membranes peroxidation: a comparison between healthy subjects and type 1 diabetic patients. J Clin Endocrinol Metab. 2004 Jun;89(6):2957-62.

13. Motta S, Letellier C, Ropert M, Motta C, Thiébault J. Protecting effect of vitamin E supplementation on submaximal exercise-induced oxidative stress in sedentary dogs as assessed by erythrocyte membrane fluidity and paraoxonase-1 activity. Vet J. 2009 Sep;181(3):288-95. doi: 10.1016/j.tvj1.2008.03.013.

14. Suematsu Y, Goto M, Park C, Nunes ACF, Jing W, Streja E, Rhee CM, Cruz S, Kashyap ML, Vaziri ND, Narayanaswami V, Kalantar-Zadeh K, Moradi H. Association of Serum Paraoxonase/Arylesterase Activity With All-Cause Mortality in Maintenance Hemodialysis Patients. J Clin Endocrinol Metab. 2019 Oct 1;104(10):4848-56. doi: 10.1210/jc.2019-00334.

15. Miljkovic M, Stefanovic A, Vekic J, Zeljkovic A, Gojkovic T, Simic-Ogrizovic S, Bogavac-Stanojevic N, Cerne D, Ilic J, Stefanovic I, Jelic-Ivanovic Z, Spasojevic-Kalimanovska V, Kotur-Stevuljevic J. Activity of paraoxonase 1 (PON1) on HDL2 and HDL3 subclasses in renal disease. Clin Biochem. 2018 Sep;60:52-8. doi: 10.1016/j.clinbiochem.2018.08.006.

16. López-Gómez JM, Portolés JM, Aljama P. Factors that condition the response to erythropoietin in patients on hemodialysis and their relation to mortality: . Kidney Int Suppl. 2008 Dec;(111):S75-81. doi: 10.1038/ki.2008.523.
17. Scott SD. Dose conversion from recombinant human erythropoietin to darbepoetin alfa: recommendations from clinical studies. Pharmacotherapy. 2002 Sep;22(9 Pt 2):160S-5S. doi: 10.1592/phco.22.14.160s.33398.

18. Shemin D, Bostom AG, Laliberty P, Dworkin LD. Residual renal function and mortality risk in hemodialysis patients. Am J Kidney Dis. 2001 Jul;38(1):85-90. doi: 10.1053/ajkd.2001.25198.

19. Vos FE, Schollum JB, Coulter CV, Doyle TC, Duffull SB, Walker RJ. Red blood cell survival in long-term dialysis patients. Am J Kidney Dis. 2011 Oct;58(4):591-8. doi: 10.1053/j. ajkd.2011.03.031.

20. Ma J, Dou Y, Zhang H, Thijssen S, Williams S, Kuntsevich V, Ouellet G, Wong MM, Persic V, Kruse A, Rosales L, Wang Y, Levin NW, Kotanko P. Correlation between Inflammatory Biomarkers and Red Blood Cell Life Span in Chronic Hemodialysis Patients. Blood Purif. 2017;43(1-3):200-5. doi: 10.1159/000452728.

21. Alonzi T, Mancone C, Amicone L, Tripodi M. Elucidation of lipoprotein particles structure by proteomic analysis. Expert Rev Proteomics. 2008 Feb;5(1):91-104. doi: 10.1586/14789450.5.1.91.

22. Hine D, Mackness B, Mackness M. Coincubation of PON1, APO A1, and LCAT increases the time HDL is able to prevent LDL oxidation. IUBMB Life. 2012 Feb;64(2):157-61. doi: 10.1002/iub.588.

23. Singh K, Singh R, Chandra S, Tyagi S. Paraoxonase-1 is a better indicator than HDL of atherosclerosis-A pilot study in North Indian population. Diabetes Metab Syndr. 2018 May;12(3):2758. doi: 10.1016/j.dsx.2017.12.006.

24. Blatter MC, James RW, Messmer S, Barja F, Pometta D. Identification of a distinct human high-density lipoprotein subspecies defined by a lipoprotein associated protein, Ki45: Identity of Ki45 with paraoxonase. Eur J Biochem. $1993 \mathrm{Feb}$ 1;211(3):871-9. doi: 10.1111/j.1432-1033.1993.tb17620.x.

25. Mackness B, Durrington P, Boulton A, Hine D, Mackness M. Serum paraoxonase activity in patients with type 1 diabetes compared to healthy controls. Eur J Clin Invest. 2002 Apr;32(4):259-64. doi: 10.1046/j.1365-2362.2002.00977.x.

26. Gbandjaba NY, Ghalim N, Hassar M, Berrougui H, Labrazi $\mathrm{H}$, Taki H, Saile R, Khalil A. Paraoxonase activity in healthy, diabetic, and hemodialysis patients. Clin Biochem. 2012 Apr;45(6):470-4. doi: 10.1016/j.clinbiochem.2012.01.005.

27. Ribeiro S, do Sameiro Faria M, Mascarenhas-Melo F, Freitas I, Mendonça MI, Nascimento H, Rocha-Pereira P, Miranda V, Mendonça D, Quintanilha A, Belo L, Costa E, Reis F, Santos-Silva A. Main determinants of PON1 activity in hemodialysis patients. Am J Nephrol. 2012;36(4):317-23. doi: $10.1159 / 000342235$.

28. Kirschbaum B. Correlation Studies of Plasma Paraoxonase Activity and Uric Acid Concentration with AAPH-Induced Erythrocyte Hemolysis in Hemodialysis Patients. Artif Organs. 2004 Mar;28(3):259-64. doi: 10.1111/j.1525-1594.2004.47294.x.

29. Shunmoogam N, Naidoo P, Chilton R. Paraoxonase (PON)1: a brief overview on genetics, structure, polymorphisms and clinical relevance. Vasc Health Risk Manag. 2018 Jun 18;14:13743. doi: 10.2147/VHRM.S165173.

30. Mahrooz A, Mackness M, Bagheri A, Ghaffari-Cherati $\mathrm{M}$, Masoumi $\mathrm{P}$. The epigenetic regulation of paraoxonase 1 (PON1) as an important enzyme in HDL function: The missing link between environmental and genetic regulation. Clin Biochem. 2019 Nov;73:1-10. doi: 10.1016/j. clinbiochem.2019.07.010. 